\title{
IR-Spectroscopy: From Industrial Applications to Novel Integrated IR-Absorption Sensors
}

Kasberger, Jürgen and Märzinger, Wolfgang

Research Center for Non Destructive Testing GmbH (RECENDT) and i-RED Infrarot Systeme GmbH

Hafenstraße 47-51, A-4020 Linz

\begin{abstract}
The research concerned with infrared spectroscopy is the first established research area at RECENDT. Starting from very basic research the work ended up in a commercially available FTNIR process spectrometer, which now is utilized to conduct applied research projects in industrial process monitoring. Additional to a reliable process spectrometer it is very important to have the entire measurement chain from the interface to the sample under investigation to the final data processing under control. Apart from the requirements in measurement technology including the spectrometer and the optical measurement setup, we will also discuss the statistical modeling established in chemistry, which is also necessary for reliable process monitoring.

For specific applications it is beneficial to have a hand-held integrated sensor for chemical analysis. Such a sensor could be designed for a very specific application with accepted less selectivity. Thus, fundamental research has been started at RECENDT dealing with an integrated IR-absorption sensor. As proof of the functional principle of the proposed sensor concept, we will show theoretical and experimental results of the waveguide based IR-absorption sensor.
\end{abstract}

\section{Introduction}

Infrared (IR) spectroscopy is a part of vibrational spectroscopy and is a widely used technique to characterize the composition of chemical substances in quantitative and qualitative manner. Even though IR-spectroscopy is a well established method in chemical labs with high fidelity as well as high flexibility, there is still a strong demand to introduce this powerful method in industrial plants for process monitoring.

The spectral information in the near infrared region is composed of single vibrational overtones as well as a mixture of overtones. In order to retrieve the concealed information out of the spectra by means of statistical modeling, a spectrometer with high spectral resolution is advantageous. Thus, FTNIR (Fourier transform near infrared process) spectroscopy is, due to the high resolution and high sensitivity, the most suitable technique for quantitative process monitoring and control. Many parameters like components concentrations in fluids, layer thicknesses and temperature can be measured in a remote way with high precision and sensitivity.

In the recent years, an FTNIR process spectrometer has been developed at the Upper Austrian Research $\mathrm{GmbH}$ (UAR), which features a robust design for industrial requirements and additionally facilitates high-speed measurements. (Since 2009 the research on non destructive testing conducted at UAR is continued by RECENDT, which was founded from the sensor technology department of the UAR.) The fairly developed process spectrometer, which is utilized for applied research projects at RECENDT, is now commercially distributed by the spin-off company i-RED Infrarot Systeme GmbH.

Having a process spectrometer available one has to take into account that a process monitoring system not only consists of the spectrometer. A process monitoring system always consists of (i) the interface to the sample, (ii) an optical connection, (iii) a spectrometer and (iv) a statistical model. Each of the parts requires a special treatment and specific know-how in order to achieve high quality prediction of an industrial process parameter.

Since the IR-spectroscopy is a well developed measurement method, no significant development at the system level can be expected anymore. The situation is somewhat different at the interface sample/IR-radiation. Since the microsystems technology is a novel technique in measurement science, the combination of microsystems technology and IR-spectroscopy could permit a measurement setup with significantly improved performance.

This contribution is divided into two sections. In section 2 we discuss all the necessary tasks in process monitoring in brevity. The interface between the IR-radiation and the sample is the first contact with a measurement system. In order to get as much information from the samples as possible, it is very important to find the best interface for a specific measurement task. In section 3 a waveguide based interface is introduce in context with an integrated IRabsorption sensor.

\section{Process monitoring}

In industrial processes, infrared optical methods are already successfully applied as non-contact measurement techniques in quality assurance and process monitoring. Through an integration of the measurement system in the production plant, the inline-process monitoring system provides real-time measurements, which furthermore can be used for process control. Since the spectral information is acquired by means of an optical method directly in the process, robust optical materials and equipment is required. Even though in the mid infrared (MIR) region the spectral information is more pronounced, non-robust optical materials like lenses and fibers hamper the utilization of MIR spectroscopic measurement systems in industrial plants. Since, e.g., Quartz fibers and NIR-lenses are very resistant in the harsh industrial environment, near infrared technology is the most suitable with respect to robustness and 
reliability. Due to the robustness and the access to molecular information, NIR-spectroscopy represents the best measurement method for process monitoring of chemical reactions.

\subsection{Process spectrometer}

In contrast to most of the spectrometers on the market, which are originally designed for laboratory purposes and adapted to industrial applications, our FTNIR process spectrometer has been optimized with respect to robustness, measurement time and cost-effectiveness from the beginning of development. Now the process spectrometer is highly developed and distributed by i-RED Infrarot Systeme $\mathrm{GmbH}$, a spin-off company of UAR.

The Fourier transform near infrared (FTNIR) process spectrometer [1] is constructed on bases of a Michelson interferometer, as schematically shown in Fig. 1. In order to obtain a robust design, a monolithic interferometer [2] is utilized in the Michelson setup. With this technology all optical components are fused with a Quartz base plate, which features a setup with minimal thermal fluctuations. A further specific feature of the optical system is the cube-corner retro reflector, which facilitates in combination with a fixed fold-back mirror a configuration of the moving mirror with minimal adjustment requirements. The entire optical setup with the specific choice of the mirror actuation leads to a rugged, shock resistant and long term stable design.

Through the interferometric effect, which occurs when the IR-beams are recombined at the beam splitter, a modulated IR-beam is detected at the integrated InGaAs-detector. Subsequently, the electric signal provided by the IR-detector - representing the interferogram - is converted into a digital signal. Due to the sophisticated electronic design, the pre-amplification and the analog to digital conversion, the digital signal features a high signal to noise ration. Furthermore, the implemented complex signal processing [1] containing two signal processing groups enables the digital processing in real time. Because of the multiple processor design, dedicated to specific tasks of signal processing and data handling, high speed measurement with fully evaluation of the spectrum with 5 single shot measurements / sec at $1.5 \mathrm{~cm}-1$ resolution or 80 single shot measurements / sec at $12 \mathrm{~cm}-1$ resolution, respectively, are possible.

The process spectrometer is fully equipped with different data interfaces in order to enable a communication with process automation systems. Even though the process spectrometer is typically controlled via remote access, the integration of a display (see Fig. 2) also facilitates a standalone operation.

\subsection{Process implementation}

Due to the close cooperation between i-RED and RECENDT, we have access to the entire hard- and software components of the utilized micro controllers and thus can adjust and reconfigure the process spectrometer for any particular application. This unique constellation enables applied research projects in inline-process monitoring. Typical projects are ranging from quality assurance for incoming products through measuring product parameters for process control to a final quality assessment of the outgoing product. A multitude of projects already have been successfully implemented in the recent years. Typically the monitoring of fluids is accomplished with specific probes (transmission, reflection, transflection) connected to the spectrometer by optical fibers, as sketched in Fig. 3. A number of manufacturers (e.g.: [4], [5]) provide fiberoptic probes with almost all kinds of materials resistant against the chemical substances under investigation. Once the appropriate probe is chosen and installed, the light is guide from a commercial halogen source via a Quartz fiber to the interface of, e.g., an immersion probe or a flow cell, where the IR-radiation interacts with the sample. Subsequently, the light is collected in a second fiber and guided to the spectrometer, which can be positioned meters way from a potential dangerous measurement position. Additionally to the immersion probe and the flow cell, which are typically use in inline application, it is also possible to utilize an ATR-unit for online analysis.

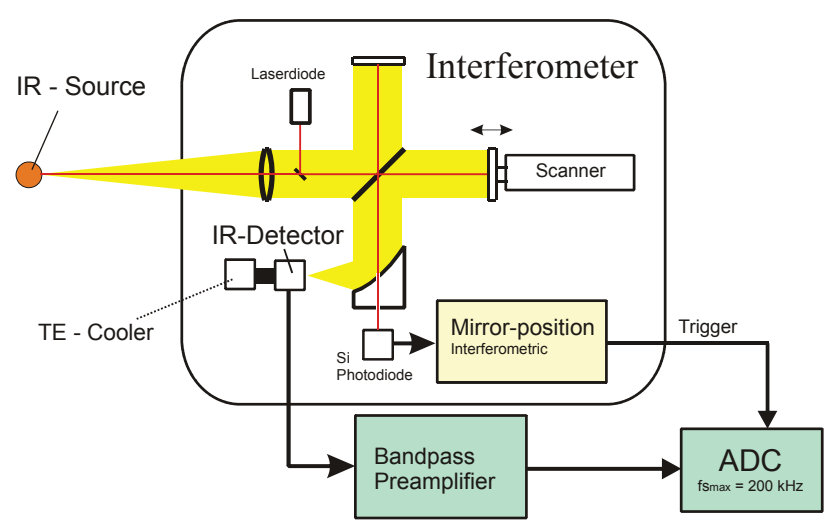

Fig. 1: Schematic of the Michelson interferometer used in the process spectrometer

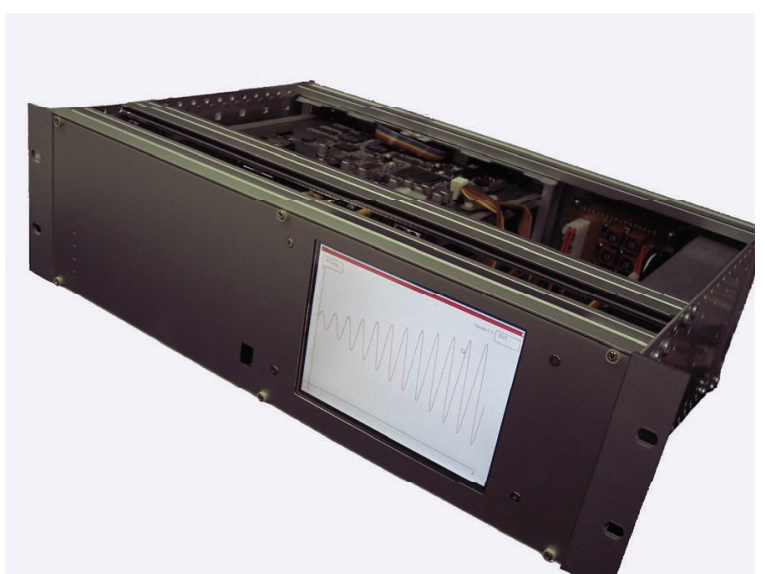

Fig. 2: Photograph of the FTNIR process spectrometer in the 19" rack mount 

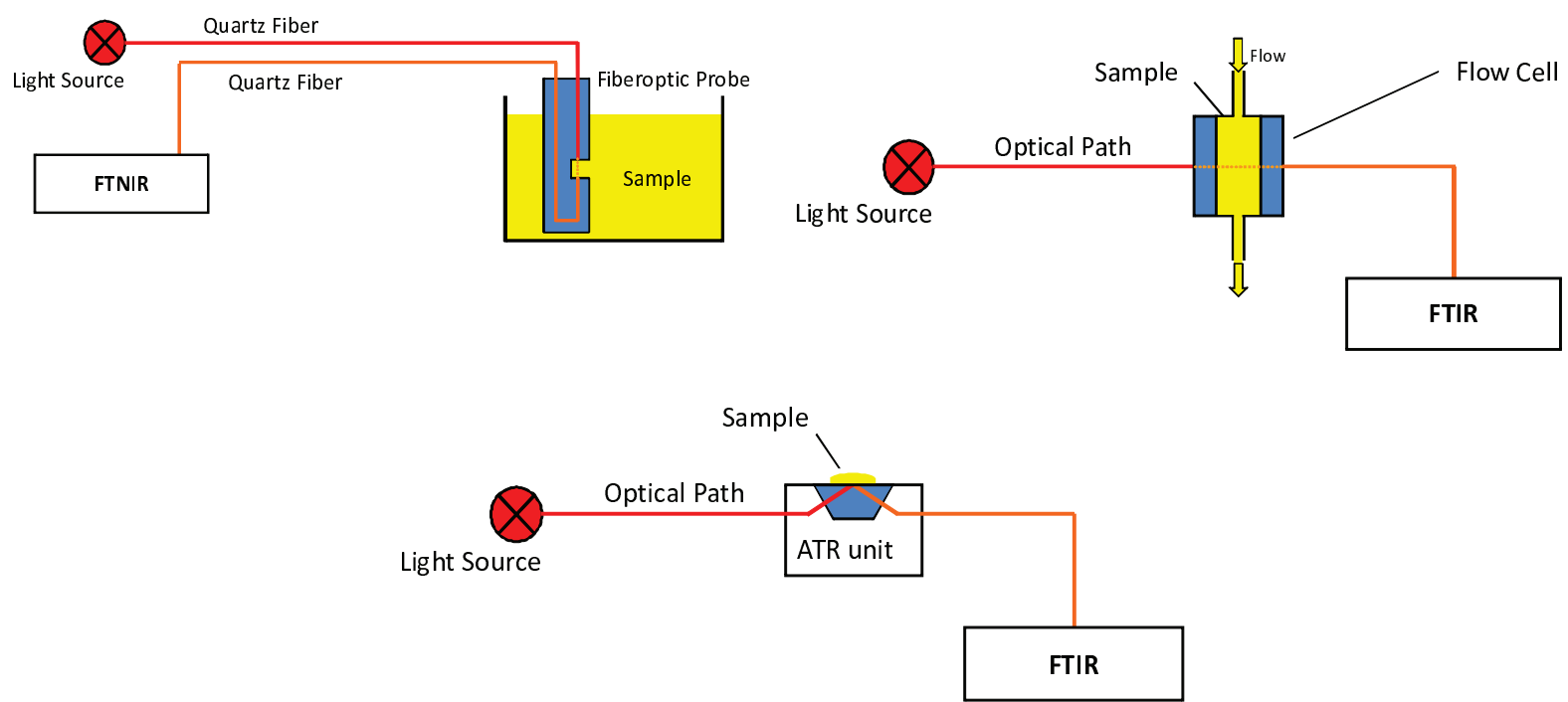

Fig. 3: Configurations with different kinds of probes for process monitoring of fluids.

Beside the commercial probes for fluids, which are provided by a number of manufacturers, we also construct fiber coupled reflection heads for surface analysis. Those reflection heads are typically composed of integrated light sources and lenses to couple the light into the fiber guiding the light to the spectrometer.

The process spectrometer is also able to trigger an optical multiplexer, which in turn facilitates a combination of different setups (Fig. 3) and measurement positions evaluated with a single spectrometer. Integrating the optical probes into the particular process plant, with the goal to obtain the best spectral information possible, is only the first step to a successful implementation of a process monitoring system.

Once the best optical setup is found, one has to care about data acquisition and an automated connection to process controlling system. Since the process spectrometer has been developed from the beginning as process spectrometer all necessary data interfaces like, e.g., LAN or CAN are available and configurable.

\subsection{Chemometrics}

In the mid infrared, where the so-called finger print region is located, the pronounced absorption peaks related to certain fundamental molecular oscillations simplify quantitative and qualitative analysis of the chemical composition. The situation is different in the NIR-range where the spectrum of a chemical mixture it composed of overtones of a certain molecule and a mixture of different molecules. Thus, a quantitative monitoring of chemical concentrations in fluids by means for FTNIR-spectroscopy, which in turn can be utilized as process parameters to control certain chemical reactions, requires multivariate data modeling (chemometrics) [6].

In statistical modeling [7] a number of linear and non-linear methods exist. Considering spectroscopic measurementst, the concentration of a certain substance in the mixture can be calculated by an exponential function given by Beer-Lambert's law [8]. Thus, through a logarithmic operation the absorbance can be calculated, which in turn results in a linear model. Therefore, in chemical statistics [8], [9] a linear method called partial least squares (PLS) has been established for a long time. Even though the PLS method is best suited for quantitative analysis when reference values are available, there also exist methods to determine concentrations when no reference analytic is feasible. For example, the multivariate curve resolution (MCR) [6] enables the decomposition of the measured spectrum into base spectra representing the spectra of the particular components in the chemical mixture. The implementation of the measurements system in an industrial process enables the collected of an almost unlimited amount of data, which is advantageous in statistical modeling. In contrast, the implementation in a real world chemical process complicates the access to the spectral data, which results in high requirements in data processing and reliable data modeling. A rather important step in (pre-)processing is the variable selection, which reduces the spectral space to the relevant areas of wavenumbers. The variable selection requires a lot of experience to identify the relevant spectral ranges in order to reduce the spurious influence of non relevant spectral information, which results in unwanted cross-correlation to effects disturbing the chemometrical model. In Fig. 4 an example is show, where a chemometric model is made for a lab concentration series for ethanol, which calculates the concentration of ethanol from the spectra shown in the plot on the left hand side of Fig. 4.

The long term experience in chemometrics and the know-how of typical requirements of real world industrial problems makes RECENDT of a qualified partner for any kind of process monitoring in fluids as well as for solids. 


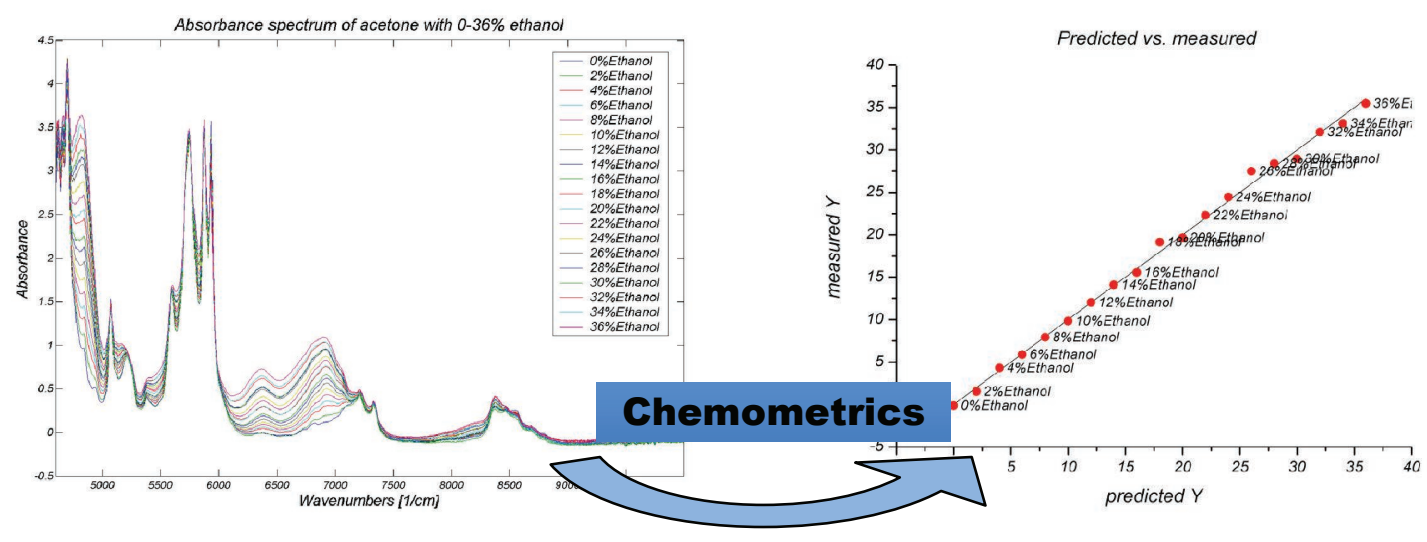

Fig. 4: Example of the transition from spectral measurements to predicated values of the concentration of ethanol.

The plot on the right hand side shows predicted values versus measured values of different concentrations of ethanol. The underlying model is calculated for the spectra show on the left hand side.

\section{Microsystems technology}

Beside commercial spectrometers, which are in general expansive and bulky laboratory measurement instruments, miniaturized spectrometers have been proposed by several authors in the recent years [10]. Most of the work on miniaturization is focused on certain parts of the entire setup like, e.g., the dispersive element. In contrast, we are working on a fully integrated system [11], as show in Fig. 5. In various targeted applications the measurement of the IR-absorption at a limited number of wavelengths is suitable to characterize a certain chemical property. For example, in order to increase the interval of scheduled maintenance of lubrication oil, e.g., in combustion engines, the quality of the deteriorated oil has to be determined. The oxidation of lubrication oil can be used as a figure of merit for the aging process. Since the aging of lubrication oil can be characterized by measuring the IR-absorption at two distinct wavelengths [12], the deterioration of lubrication oil has been selected as example application. This application is suitable for initial investigations, due to the fact that only two distinct wavelengths $\left(\lambda_{1}=5.08 \mu \mathrm{m}\right.$ and $\lambda_{2}$ $=5.85 \mu \mathrm{m}$ ) are required with low requirement in spectral resolution. However, having sophisticated micro-structuring processes under control enables the fabrication of photonic micro-structures with features beating conventional macro-structures. For example, as an alternative to the targeted fully integrated system, one could also utilize the waveguide structure as a substitute of a conventional ATR (attenuated total reflection) - element, featuring outstanding sensitivity due to micro-structuring.

\subsection{Design of an integrated IR-absorption sensor}

Regarding our efforts in microsystems technology, we aim at a fully integrated absorption sensor, as sketched Fig. 5, based on IR-absorption in the evanescent field of an integrated mono-mode waveguide utilizing thermally generated and detected IR-radiation. The thermal radiation excitation and detection was selected due to the targeted mid-IRregion. Even though non thermal elements exhibit outstanding features like, e.g, the high sensitivity of cryogenic cooled detectors, those non-thermal elements are not suitable for integration into a mirco-sensor due to the associated comparatively large dimensions.

As shown in [11], for a properly designed mono-mode waveguide the attenuation coefficient of the guided optical wave - attenuated due to the absorption of the sample in the evanescent field - is comparable to the attenuation of an IR-beam in a transmission measurement in the bulk sample. Achieving the best sensitivity of the mono-mode waveguide requires that the refractive index of the sample is smaller than the refractive index of the substrate. This requirement and the targeted mid-IR-range, where only a limited number of materials are transparent, results in a waveguide composed of $\mathrm{SiN}$ and $\mathrm{MgF}_{2}$.

Considering the performance of the entire sensor system one also has to take into account the coupling properties of the grating couplers [13] as well as the different propagation constants of the fundamental transverse electric (TE) and transverse magnetic (TM) mode [14]. Both the coupling and the different propagation constants of the fundamental modes result in a reduced spectral resolution of the concept shown in Fig. 5. However, as already mentioned above, the waveguide can also be used as an IR-transducer in combination with an IR-spectrometer.

\subsection{Experimental results}

In order to characterize the proposed mono-mode waveguide as an IR-absorption element, we have fabricated a waveguide structure including two grating couplers with the materials discussed above. The concept of the fully integrated absorption sensor, as shown in Fig. 5, utilizes the angular coupling characteristics of grating couplers for the spectral separation of the measurement wavelength. To characterize the mere waveguide as absorption element, we have built up a measurement setup, where the spectral measurement is done with an IR-spectrometer [15]. To characterize the waveguide prototype, comprising the input grating coupler, the waveguide and the output grating coupler, the total transmission through the sample is measured for a certain fixed coupling angle. In order to 


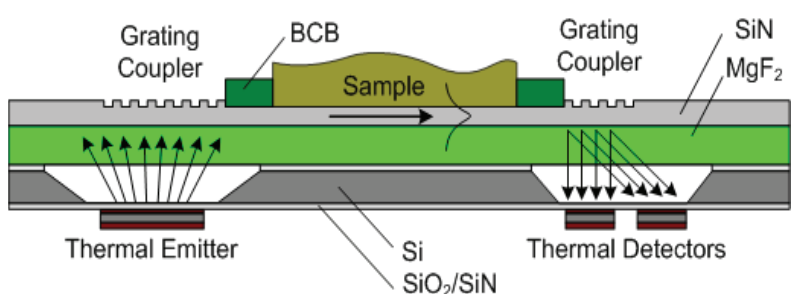

Fig. 5: Concept of a fully integrated sensor system for the mid IR-range. Two grating couplers are utilized 1.) to couple broadband IR-radiation into the waveguide and 2.) subsequently for spectral separation when coupling out of the waveguide.

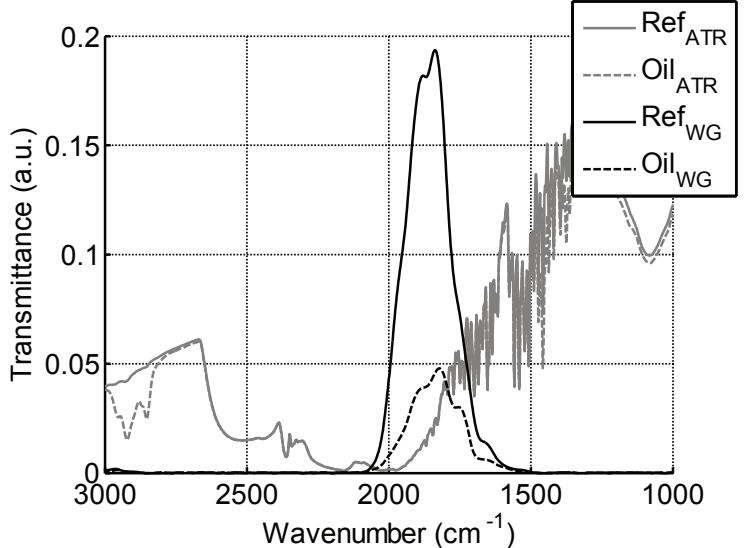

Fig. 6: Total transmission through the gratings and the waveguide of reference (i.e., no oil loaded on the waveguide) and oil measurements. Here a commercial ATR-element and the investigated waveguide prototypes are compared regarding the absorption in the evanescent field of devices.

demonstrate the sensitivity of the propose mono-mode waveguide, measurements obtained with a commercial ATRelement (three reflections) are compared to those obtained with our waveguide structure, as shown in Fig. 5. For both evanescent field absorption elements (ATR and mono-mode waveguide) a reference measurement as well as the corresponding oil measurement, where the oil is loaded on top of the waveguide without changing the optical setup, are depicted. The decrease in transmission for the oil measurement compared to the corresponding reference measurement, which is only recognizable for the mono-mode waveguide (Fig. 6), confirms the fundamental sensitivity of the proposed mono-mode waveguide structure. With the obtained sensitivity, this measurement setup can also be applied for monitoring the deterioration of the investigated lubrication oil, as demonstrated in [15].

In [11] it has been shown that the concept, depicted in Fig. 5, can be utilized as oil aging sensor. Instead of using two IR-detectors, as shown in Fig. 5, a single element has been positioned with a motorized translation stage to scan the spectral range in the used lab setup.

\subsection{Increased power density of the guided wave}

In the mid-IR-range the available power is limited, which results in high requirements with respect to the signal to noise ration. Since we utilize in the proposed fully integrated sensor thermal IR-detectors with less sensitivity than cryogenic cooled IR-detectors, we attempt to improve the sensitivity by means of exploiting the features of photonic structures, as shown in Fig. 7.

A thermal IR-source simply represents a heated body emitting IR-radiation described by Planck's law. In case of a thermal emitter the power emitted by the surface is proportional to the surface area and the temperature. Thus, increasing the width (coupling area) of a grating coupler increases the energy coupled into the waveguide but not the energy density. A taper structure on the other hand increased the energy density (see Fig. 8) but not the total guided energy. In consequence, introducing a taper structure, as shown in Fig. 7, enables the implementation of a wide

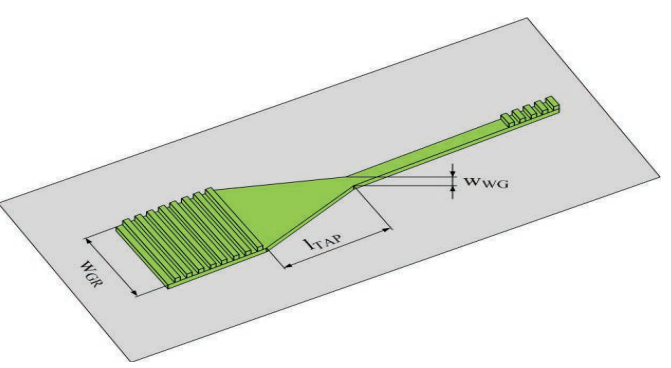

Fig. 7: Sketch of an integrated taper structure of a $\mathrm{SiN}$-waveguide on a $\mathrm{MgF}_{2}$-substrate. The insertion of the taper structure features an improved energy density resulting in a better signal to noise ratio.

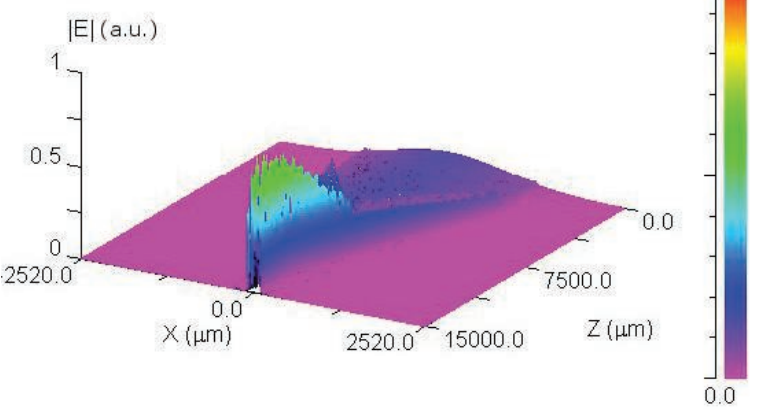

Fig. 8: Numerical simulation of the taper structure using the beam propagation method (BPM, RSoft).

The calculations have been carried out in a cooperation with the Center for Surface- and Nanoanalytics, Johannes Kepler University Linz. 
grating coupler - yielding high energy in a wide waveguide - in conjunction with a small waveguide guiding the IRradiation. This configuration can be utilized in combination with a small IR-detector obeying less noise than larger detectors.

\section{Conclusion}

Vibrational spectroscopy is a powerful method in characterizing chemical substances regarding their quantitative and qualitative composition. However, the application of vibrational spectroscopy in the different fields from process monitoring to lab applications requires an interdisciplinary team covering chemistry, physics, measurement technology and even statistics. Even though the available measurement technology including probes, fibers and spectrometers facilitates high quality analysis, the interface between the sample and the IR-radiation still offers a lot of improvement. Especially the emerging microsystem technology shows features beating the conventional measurement equipment. The investigations carried out so far, have show promising theoretical and experimental result. However, there is still a lot of work to do in order the understand and exploit the full potential of photonic structures in infrared technology.

\section{Acknowledgment}

This work was supported by the European Regional Development Fund (EFRE) in the framework of the EU program Regio 13 and the federal state Upper Austria. We would also like the thank Ahmad Saeed (Institute of Semiconductor and Solid State Physics, Johannes Kepler University Linz) and Thomas Fromherz (Institute of Semiconductor and Solid State Physics, Johannes Kepler University Linz) for supporting the work related to microsystems technology.

\section{References}

[1] P.M. Hintenaus, W. Märzinger, An Infrared Spectrometer for Process Monitoring I, Spectroscopy, Proc. IECON, 2007.

[2] Z. Bleier, C. Brouillette, R. Carangelo, A Monolithic Interferometer for FT-IR Spectroscopy, Spectroscopy 14(10), 1999

[3] P.M. Hintenaus, W. Märzinger, H.Pöll, An Infrared Spectrometer for Process Monitoring II, Chemometry and Automatization, Proc. EESMS, 2010.

[4] http://www.hellma-analytics.com

[5] http://www.solvias.com/

[6] R. Kessler, Prozessanalytik, Wiley-VCH, 2006.

[7] T. Hastie, R. Tibshirani, J. Friedman, The Elements of Statistical Learning: Data Mining, Inference, and Prediction., Springer, 2009, online: http://www-stat.stanford.edu/ tibs/ElemStatLearn.

[8] J. N. Milller, J.C. Miller, Statistics and Chemometrics for Analytical Chemistry, Pearson, 2000.

[9] M. Otto, Chemometrics: Statistics and Computer Application in Analytical Chemistry, Wiley-VCH, 1999.

[10] R. F. Wolffenbuttel, State - of - the - Art in Integrated Optical Microspectrometers, IEEE Trans. on Instr. and Meas., vol. 53/1, pp. 197-202, 2004.

[11] J. Kasberger, et al., Miniaturized integrated evanescent field IR-absorption sensor: Design and experimental verification with deteriorated lubrication oil, Vib. Spectrosc. (2011), doi:10.1016/j.vibspec.2011.01.003.

[12] A. Agoston, C. Ötsch, J. Zhuravleva, B. Jakoby, An IR-Absorption Sensor System for the Determination of Engine, Proceedings IEEE Sensor 2004, pp.463-466.

[13] J. Kasberger, B. Jakoby, Thermische Kopplungseigenschaften eines Gitterkopplers als zentrales Element eines IR-Absorptionssensors, Tech. Messen vol. 75, 2008.

[14] J. Kasberger, B. Jakoby, Design of a Novel Fully Integrated IR - Absorption Sensor System, Proc. IEEE Sensors, 2007.

[15] J. Kasberger, P. Rauter, T. Fromherz, B. Jakoby, A Slab Waveguide Sensor for a Fully lintegrated IRAbsorption Sensor System - Theoretical and Experimental Verification Proc. Transducers (2009) 999-1002.

[16] J. Kasberger, A. Saeed, B. Jakoby, K. Hingerl, „Improvement of an Evanescent Field IR-Absorption Sensor by utilizing a Photonic Taper Structure, Proc. Eurosensors XXIV, 2010. 